\title{
CELULAS SANGUINEAS DE SCHROEDERICHTHYS CHILENSIS (GUICHENOT 1848) (ELASMOBRANCHII, SCYLIORHINIDAE): LA SERIE BLANCA
}

\section{BLOOD CELLS OF THE SCHROEDERICHTHYS CHILENSIS (GUICHENOT 1848): THE LEUKOCYTES (ELASMOBRANCHII, SCYLIORHINIDAE)}

\author{
Ariel Valenzuela ${ }^{1}$, Ciro Oyarzún ${ }^{2} \&$ Víctor Silva $^{3}$ \\ ${ }^{1}$ Programa de Doctorado en Ciencias Biológicas, Facultad de Ciencias Naturales y Oceanográficas, Departamento \\ de Oceanografía, Laboratorio de Piscicultura. Universidad de Concepción. P.O.Box 160-C. Concepción, Chile. \\ E-Mail: avalenz@udec.Cl \\ ${ }^{2}$ Facultad de Ciencias Naturales y Oceanográficas, Departamento de Oceanografía. Universidad de Concepción. \\ ${ }^{3}$ Facultad de Farmacia, Departamento de Bioquímica Clínica e Inmunología. Universidad de Concepción.
}

\section{RESUMEN}

La Ictiohematología es una herramienta de uso común con fines clínicos en peces de importancia económica, con un menor aporte en la comprensión de la fisiología del organismo en relación con su medio. En este contexto y frente a la escasa información publicada de aspectos hematológicos de condrictios, se propuso caracterizar los leucocitos de la pintarroja Schroederichtys chilensis y determinar los valores de referencia cuantitativos. Se obtuvo sangre de pintarrojas capturadas en la Bahía Coliumo (VIII Región, Chile). En extensiones teñidas con May Grünwald y Giemsa se caracterizó los siguientes leucocitos: linfocitos, granulocitos eosinófilos, granulocitos polimorfonucleares heterófilos, monocitos, blastos y trombocitos. Los leucocitos, en general, presentaron características similares a las descritas en otros peces, destacando la elevada presencia de eosinófilos con gránulos prominentes. Cuantitativamente, se encontraron en el siguiente orden: trombocitos $(40-50 \%)>$ eosinófilos $(15-25 \%)>\operatorname{linfocitos}(18-25 \%)>$ heterófilos $(5-9 \%)>$ monocitos $(0-1 \%)=$ blastos $(0-1 \%)$. En comparación con otros peces, la eosinofilia (la más elevada descrita en peces), produce una distorsión de la fórmula leucocitaria, posiblemente por la presencia de trypanosoma humboldti en la sangre. Los parámetros hematológicos informados son un aporte al conocimiento de la especie y servirán como base comparativa para futuras aplicaciones.

Palabras claves: Schroederichthyes chilensis, Hematología, leucocitos, eosinofilia, tripanosoma, Chile.

\begin{abstract}
Ichthyohematology is a common tool in the clinic analysis of economically important fish, and used less to understand fish physiology in relation to the environment. In this context and because of the scarce information about the condrictian hematology, we characterize the leukocytes of the Chilean catshark Schroederichtys chilensis and determine the quantitative reference values. Blood was obtained from fish caught in Coliumo Bay (VIII Región, Chile). Slides were stained with May Grünwald Gimsa. Lymphocytes, eosinophil granulocytes, heterophils granulocytes, monocytes, blasts and thrombocytes were characterized. The leukocytes show similar characteristics to other fish, emphasizing the high number of eosinophils with big granules. Quantitatively the rank order is: thrombocytes (40-50\%) > eosinophils (15$25 \%)>$ lymphocytes $(18-25)>$ heterophils $(5-9 \%)>$ monocytes $(0-1 \%)=$ blasts $(0-1 \%)$. Comparing with other fish, the catshark eosinophilia (the highest reported among fishes) produces a distortion in the differential leukocyte count, probably due to the presence of Trypanosomes in the blood. The hematological parameters here reported are important for the species knowledge and will be useful as a comparative base for future studies.
\end{abstract}

Keywords: Schroederichthys Chilensis, Hematology, leukocytes, eosinophilia, Trypanosome, Chile. 


\section{INTRODUCCION}

La Ictiohematología puede ser definida en términos generales, como una disciplina que estudia la sangre de los peces; sin embargo, en términos prácticos esta especialidad estudia las células sanguíneas morfológica, bioquímica y funcionalmente como también los órganos hematopoyéticos, las enfermedades relacionadas con ellos y cualquier fenómeno o patología que relacione las células y/o sus órganos productores (Williams et al. 1991). De este modo, las variaciones de los parámetros hematológicos como hematocrito, leucocrito, recuentos celulares y concentración de hemoglobina pueden, entre otros, ser utilizados como indicadores de contaminación (Zbanyszek \& Smith 1984, Hantonella 1998, Whali 2002). A su vez, los parámetros hematológicos están siendo utilizados como indicadores fisiológicos de disfunción orgánica por estrés (Wedemeyer et al. 1990). Así, por ejemplo, la tensión de oxígeno tiene implicancias directas en la regulación de la eritropoyesis de modo que la hipoxia produce, como respuesta aguda, la liberación de eritrocitos por contracción esplénica, causando aumento de policromatófilos (eritrocitos inmaduros) en circulación (Kita \& Itazawa 1989, Cerda 1994, Valenzuela et al. 2002); por otra parte, el estrés crónico produce leucopenia y cambios en la fórmula leucocitaria como linfopenia, monocitopenia y neutrofilia (Wedemeyer etal.1990, Donaldson 1981).

La pintarroja (Schroederichthys chilensis,
Guichenot, 1848) tiene una distribución en el Pacífico sur desde Ancón (Perú) hasta el extremo austral de Chile (Compagno 1984), es ovípara y de alimentación carnívora habitando en las cercanías de la plataforma continental (Fariña \& Ojeda 1993); su único uso conocido hasta el momento, es como carnada para la captura de jaibas.

Hematológicamente, los peces Cartilaginosos se caracterizan por presentar valores de hematocrito, recuentos de eritrocitos y hemoglobina menores a los encontrados en peces óseos (Wilhelm et al.1992) y dentro de los cartilaginosos, los escualimorfos tenderían a presentar valores mayores que los bátidos (Larsson et al. 1976). Hasta el momento, los antecedentes hematológicos publicados para S. chilensis se remiten a la descripción de la ultraestructura de granulocitos (Morillas 1981a, 1981b).

Sobre $S$. chilensis se ha desarrollado un creciente interés por utilizarlo como bioindicador de contaminación química en la Octava Región. Sin embargo, el conocimiento hematológico de Chondrichthyes chilenos es muy escaso, lo cual crea la necesidad de aumentar el conocimiento de esta especie, obteniendo datos que puedan ser utilizados en su mantención tanto en su ambiente natural como en cautiverio y, además, servir como referencia para futuras investigaciones experimentales en el campo de la Hematología Comparada y otras disciplinas. Así, en este trabajo informamos los aspectos morfológicos y cuantitativos de leucocitos de $S$. chilensis para que sirvan como parámetros de la función, del tipo celular y del estado del animal.

TABLa I.- Datos biometricos de S. chilensis.

TABLE I.- Biometric data of the S. chilensis

\begin{tabular}{clccc}
\hline Año & Medición & Promedio General $^{\circ}$ & Promedio Hembras & Promedio Machos \\
\hline \multirow{2}{*}{1998} & Peso $^{\text {a }}$ & $257.2(129.5)$ & $241.6(100.6)$ & $279.0(173.0)$ \\
& Longitud $^{\text {b }}$ & $43.3(5.3)$ & $42.7(4.6)$ & $44.2(6.6)$ \\
2000 & Peso & $184.8(85.4)$ & $200.1(100.2)$ & $149.0(40.5)$ \\
& Longitud & $38.5(4.7)$ & $39.0(5.6)$ & $37.3(1.5)$ \\
2001 & Peso & $194.2(94.2)$ & $206.9(120.7)$ & $173.2(29.0)$ \\
& Longitud & $39.0(5.2)$ & $38.8(6.8)$ & $39.3(1.5)$ \\
2002 & Peso & $202.2(91.9)$ & $191.5(110.7)$ & $218.3(91.6)$ \\
& Longitud & $42.9(8.7)$ & $40.0(7.2)$ & $47.3(11.7)$ \\
\hline
\end{tabular}

a) en gramos b) en $\mathrm{cm}$ c) promedio (desviación estándar) 
Gayana 67(1), 2003

\section{MATERIALES Y METODOS}

Se analizaron hematológicamente 38 pintarrojas capturadas en la bahía de Coliumo (36³2'S; 72 ${ }^{\circ} 57^{\prime} \mathrm{W}$ ) en septiembre de 1998 , diciembre de 2000 , noviembre de 2001 y enero de 2002; los datos biométricos son presentados en la Tabla I. Los individuos fueron mantenidos en estanques para su aclimatación a una densidad de $5,6 \mathrm{~K} / \mathrm{m}^{3}$ con flujo constante de agua y alimentados periódicamente con trozos de pescado y/o moluscos frescos. Las pintarrojas capturadas a partir del 2000 fueron alimentadas con pellet para salmonídeos.

La sangre fue obtenida por punción caudal de animales anestesiados con etil p-aminobenzoato (BZ-20), siguiendo los procedimientos sugeridos por Campbell \& Murru (1990), y colocada en tubos Eppendorf con EDTA. Las extensiones sanguíneas (dos por cada pez) fueron realizadas de inmediato, dejándolas secar a temperatura ambiente; posteriormente, fueron teñidas con May-Grünwald Giemsa. El conteo de células se realizó en sangre diluida 1:100 con la solución de Rees-Ecker en cámara de Neubauer (Campbell 1988; Campbell \& Murru 1990).

La fórmula leucocitaria se realizó sobre la base de 100 células. La descripción citológica se realizó con microscopio óptico (Carl Zeiss) midiendo, con micrómetro ocular, el diámetro mayor de las células. La comparación de las formulas leucocitarias entre machos y hembras y entre los distintos años de muestreo se realizó por medio de la prueba t-Student. Estos análisis y la estimación del limite de confianza del $95 \%$ para el promedio de cada tipo celular (Valores de referencia) se realizaron con el programa Statisca 5.1.

TABLa II. Descripción de las células sanguíneas de $S$. chilensis (n= número de células).

TABLE II. Blood cells description of S.chilensis. $(\mathrm{n}=$ number of measured cells).

\begin{tabular}{|c|c|c|c|c|}
\hline Tipo celular & $n$ & núcleo & Citoplasma & Diámetro $(\mu m)^{\mathrm{a}}$ \\
\hline $\begin{array}{l}\text { 1.- Trombocito } \\
\text { maduro, } \\
\text { alargado }\end{array}$ & 15 & $\begin{array}{l}\text { Central, cromatina semejante a } \\
\text { eritrocitos, ocupa aprox. el } 50 \% \\
\text { de la célula. }\end{array}$ & $\begin{array}{l}\text { Citoplasma poco } \\
\text { abundante, azurófilo } \\
\text { pálido, sin gránulos. }\end{array}$ & $13.6( \pm 3,6)$ \\
\hline 2.- Linfocito & 25 & $\begin{array}{l}\text { Excéntrico ocupa sobre el } 70 \% \\
\text { de la célula, color violeta } \\
\text { redondo y de cromatina } \\
\text { granulosa. }\end{array}$ & $\begin{array}{l}\text { Generalmente redondo, } \\
\text { basófilo oscuro sin } \\
\text { gránulos. }\end{array}$ & $7.4( \pm 0,5)$ \\
\hline 3.- Heterófilo & 15 & $\begin{array}{l}\text { Excéntrico ocupando } 20-30 \% \text { de } \\
\text { la célula, color violeta, redondo } \\
\text { con finos granos y generalmente } \\
\text { con lóbulos. }\end{array}$ & $\begin{array}{l}\text { Redondo, fondo } \\
\text { citoplasmático celeste } \\
\text { con finas granulaciones. }\end{array}$ & $13.0( \pm 2.7)$ \\
\hline 4.- Eosinófilo & 20 & $\begin{array}{l}\text { Excéntrico ocupando } 20-30 \% \text { de } \\
\text { la célula, color violeta, con } \\
\text { lóbulos. }\end{array}$ & $\begin{array}{l}\text { Redondo, grandes } \\
\text { gránulos naranja en } \\
\text { fondo celeste. }\end{array}$ & $15,0( \pm 3,6)$ \\
\hline 5.- Monocito & & $\begin{array}{l}\text { Generalmente reniforme, } \\
\text { semiexcéntrico ocupa más del } \\
50 \% \text { de la célula. }\end{array}$ & $\begin{array}{l}\text { Irregular o redondo de } \\
\text { color azul gris. }\end{array}$ & No medido \\
\hline 6.- Blastos & 2 & $\begin{array}{l}\text { Abundante, central, cromatina } \\
\text { poco densa. }\end{array}$ & $\begin{array}{l}\text { Pequeña cantidad, sólo } \\
\text { rebordes de intensa } \\
\text { basofilia, sin gránulos. }\end{array}$ & 14.0 \\
\hline
\end{tabular}

a) promedio \pm desviación estándar 

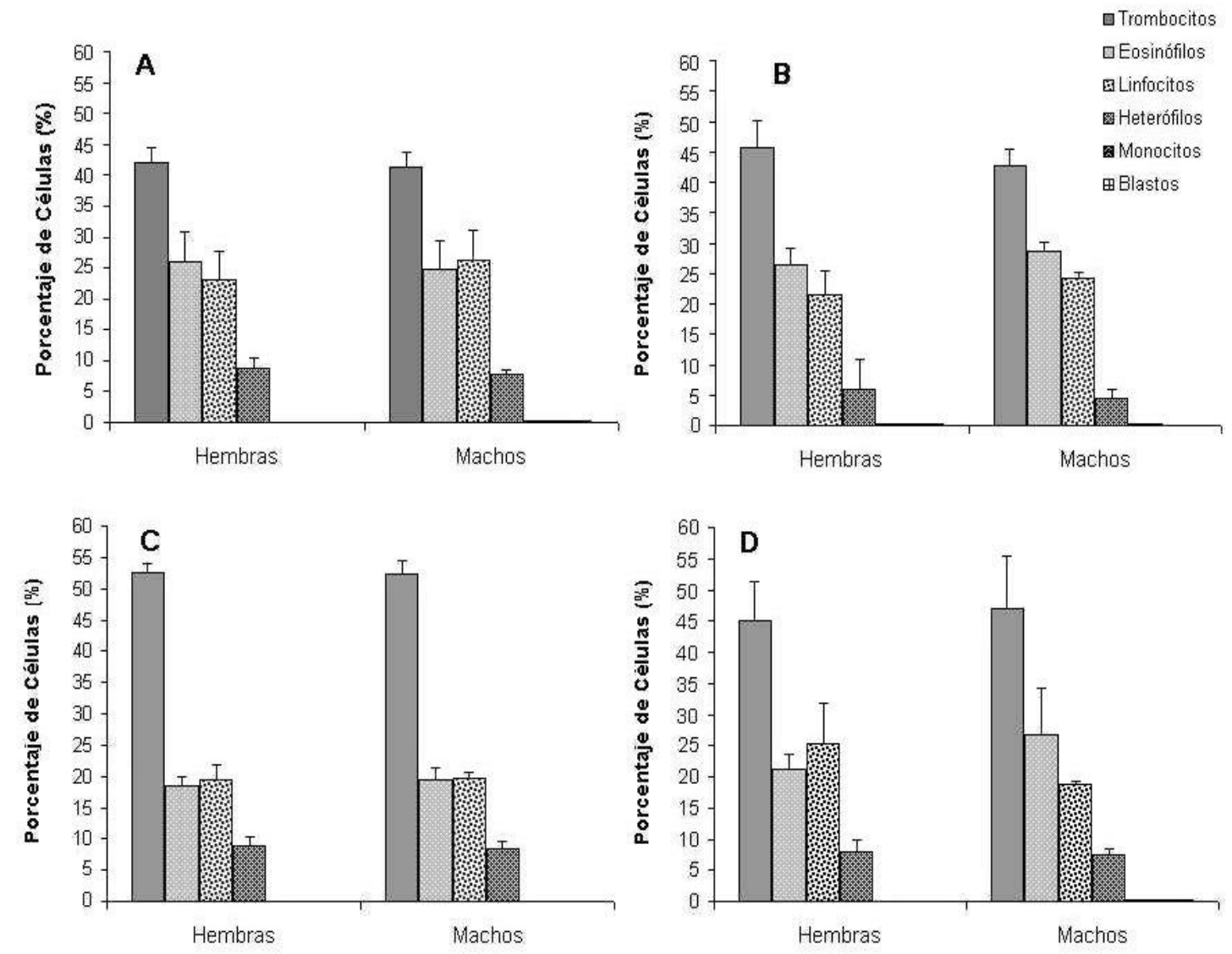

Figura 1. Formula Leucocitaria de S. chilensis en valores relativos (\%), por sexo y por año. A) 1998. B) 2000. C) 2001. D) 2002 .

Figure 1. Differential leukocyte counts in S. chilensis separated by sex and years. A) 1998. B) 2000. C) 2001. D) 2002.

\section{RESULTADOS Y DISCUSION}

Aspectos citológicos: En las extensiones de sangre periférica de $S$. chilensis se identificaron 6 tipos celulares cuyos valores porcentuales se expresan en la Figura1. Los tipos celulares encontrados son similares a los descritos en otros peces, sólo que en sangre periférica de $S$. chilensis no se observaron basófilos. A continuación se describe las características fundamentales de las células sanguíneas de S.chilensis resumidas en la Tabla II.

Trombocitos

Este tipo celular, cuya principal función es mante- ner la hemostasis, se visualizó principalmente como células alargadas (largo promedio de $13.6 \mathrm{~mm}$ ), con poco citoplasma distribuido en uno o ambos lados y sin gránulos (Fig. 2a). En ocasiones estas células también aparecen con núcleo oval, siendo muy semejante a un linfocito.

\section{Leucocitos}

Monocitos: Se encontraron ocasionalmente $(<1 \%)$; su descripción general es la descrita en la Tabla II y son fácilmente confundibles con algunos blastos (Fig. 2b) (células indiferenciadas).

Linfocitos: Constituyen el tipo leucocitario 
Gayana 67(1), 2003
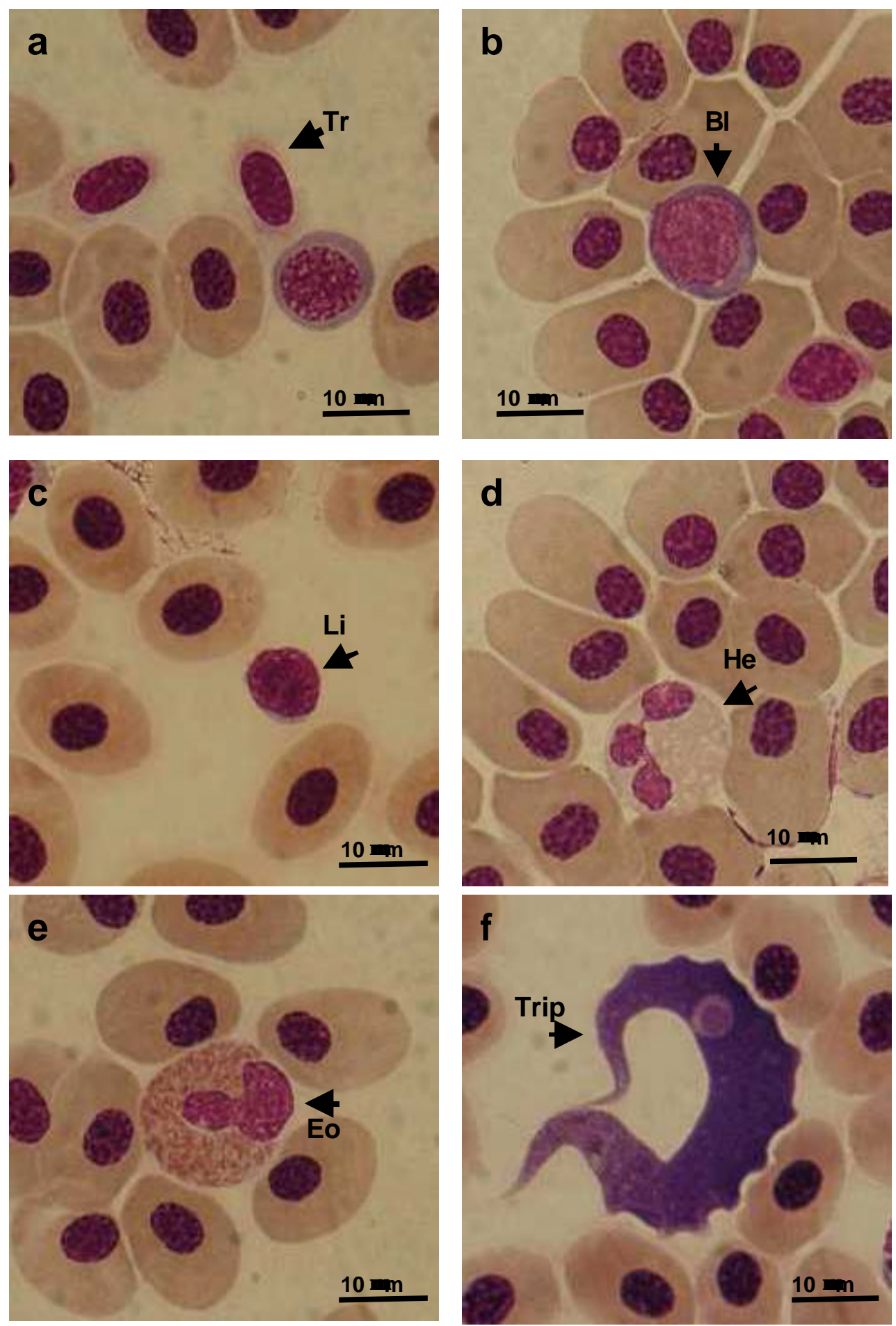

Figura 2. Leucocitos de S. chilensis. A) Trombocitos (Tr) B) Blasto (Bl) c) Linfocito (Li) d) Heterófilo (He) e) Eosinófilo (Eo) f) T. humboldti (Tri). Tinción May Gründwald Giemsa.

Figure 2. Leukocytes of the S. chilensis. a) Trombocyte (tr) b) Blast cell (Bl) c) Lymphocyte (Li) d) Heterophil (He) e) Eosinophil (Eo) f) T. humboldti (Tri). May Gründwald Giemsa stain. 
Leucocitos de S. chilensis: Valenzuela, A. ET AL

TABLA III. Valores de referencia de la formula leucocitaria de S.chilensis en bahia Coliumo. Distribución anual.

TABLE III. Reference values of the differential leukocyte count for S. chilensis in Coliumo Bay.

\begin{tabular}{|c|c|c|c|}
\hline Año & Leucocito & Promedio $\left(10^{9} / 1\right)$ & $\begin{array}{l}\text { Limite de Confianza } \\
(95 \%)\end{array}$ \\
\hline \multirow{6}{*}{1998} & Eosinófilos & 2.90 & $2.07-3.72$ \\
\hline & Linfocitos & 2.65 & $2.01-3.29$ \\
\hline & Heterófilos & 0.94 & $0.71-1.18$ \\
\hline & Monocitos & 0.02 & $0.001-0.03$ \\
\hline & Blastos & 0.01 & $0.0-0.03$ \\
\hline & Trombocitos & 4.60 & $3.59-5.60$ \\
\hline \multirow{6}{*}{2000} & Eosinófilos & 3.05 & $2.08-4.01$ \\
\hline & Linfocitos & 2.51 & $1.68-3.34$ \\
\hline & Heterófilos & 0.58 & $0.30-0.86$ \\
\hline & Monocitos & 0.02 & $0.001-0.04$ \\
\hline & Blastos & 0.01 & $0-0.03$ \\
\hline & Trombocitos & 5.02 & $3.45-6.59$ \\
\hline \multirow{7}{*}{2001} & Eosinófilos & Machos 2.23 & $0.25-4.22$ \\
\hline & & Hembras 2.28 & $1.30-3.26$ \\
\hline & Linfocitos & 2.29 & $1.79-2.79$ \\
\hline & Heterófilos & 1.06 & $0.71-1.40$ \\
\hline & Monocitos & 0.01 & $0-0.01$ \\
\hline & Blastos & 0.01 & $0-0.01$ \\
\hline & Trombocitos & 6.27 & $4.69-7.85$ \\
\hline \multirow{6}{*}{2002} & Eosinófilos & 2.77 & $1.58-3.95$ \\
\hline & Linfocitos & 2.6 & $1.50-3.70$ \\
\hline & Heterófilos & 0.99 & $0.33-1.65$ \\
\hline & Monocitos & 0.01 & $0-0.03$ \\
\hline & Blastos & 0.01 & $0-0.03$ \\
\hline & Trombocitos & 5.72 & $2.19-9.25$ \\
\hline
\end{tabular}

mayoritario en teleósteos; sin embargo en $S$. chilensis representan un 18 a $25 \%$ (Fig. 1), valores cercanos al 29\% informado por Morillas (1981a); estas células son de forma irregular y el núcleo ocupa gran parte del citoplasma (Fig. 2c); morfológicamente son similares a los descritos en otros teleósteos (Ellis 1977, Mahajan \& Dheer 1980,
Valenzuela etal. 1999) y en Squalus.acanthias, sólo que en esta especie son el tipo leucocitario más representativo (Sherburne 1974).

Heterófilos: Corresponden a granulocitos polimorfonucleares (PMN) heterófilo de $13 \mathrm{~mm}$ de diámetro, generalmente con núcleo lobulado 
(Fig.2d) y citoplasma con finas granulaciones casi imperceptibles. Cuantitativamente, la población estudiada presentó valores entre 0.58 y $1.06 \times 10^{9}$ células/l (Tabla III) lo que se aproxima a un 5 - 10 $\%$ del total de leucocitos, bastante menos de lo informado por Morillas (1981a).

Eosinófilos (Fig. 2e): Son células cuya función en peces no es completamente clara (Ellis 1977); sin embargo, tienden a encontrarse en zonas como aparato digestivo, piel y filamentos branquiales (Powell et al. 1990; Barnett et al. 1996) relacionándose con la estimulación antigénica e infecciones parasitarias. Estas células en $S$ chilensis tienen un diámetro promedio de $15 \mathrm{~mm}$, núcleo lobulado y gránulos citoplasmáticos anaranjados de mayor tamaño que en mamíferos. Si bien estas células se encuentran descritas para peces, su frecuencia interespecies es baja; así, por ejemplo se describen en Misgurnus anguillicaudatus (Ishizeki et al. 1984), Oreochromis mossambicus (Barnett et al. 1996) y Eleginops maclovinus (Valenzuela et al. 1999), todos ellos en porcentajes menores al $10 \%$.

La eosinofilia que presenta $S$. chilensis $(15$ a $25 \%$ ) es inusual en peces; sin embargo, la presencia del protozoo Trypanosoma humboldti (Fig. 2f) en la sangre de casi todos los individuos analizados (87\% de prevalencia), podría relacionarse, al igual que en mamíferos, con la respuesta eosinofílica a infecciones parasitarias (Williams 1991). Tripanosoma humboldti fue descrito en $S$. chilensis en 1987 y sería uno de los 6 tipos de tripanosomas en elasmobranquios, de los cuales sólo uno corresponde a tiburones (Morillas et al. 1987), Sin embargo estos investigadores, aún cuando realizaron la descripción de los eosinófilos y del parásito no establecieron relaciones entre ambos. Khan (1977) al infectar individuos de Gadus morhua con trypanosoma murmanensis sólo describió una seudoanemia, sin referirse a una respuesta eosinofílica. A su vez, Valenzuela et al. (1999) describió un 5\% de eosinófilos en E. maclovinus, uno de los mayores porcentajes detectados en peces, sin observar presencia evidente de parásitos sanguíneos; todo lo cual permite postular que la eosinofilia de $S$. chilensis también podría ser propia de la especie y no secundaria a la parasitosis.

Al comparar las formulas leucocitarias entre machos y hembras no se encontró diferencias significativas entre ambos sexos $(p>0.05)$ a excepción del año 2001 en el cual las hembras presentaron más eosinófilos (Tabla III). Por otra parte, en el tiempo, se encontró diferencias significativas en la cantidad de eosinófilos entre los años 1998 - 2001 y el 2000 - 2001, (al igual que los trombocitos). En ambos casos, las diferencias son sólo en términos relativos (porcentuales) y no así en valores absolutos que son los que realmente importan cuando se relacionan variaciones cuantitativas con alguna patología. Los heterófilos, en cambio, presentaron diferencias tanto en términos relativos como en valores absolutos entre 1998 y el 2000. Por esta razón, los valores de referencia entregados, no consideran las diferencias sexuales a excepción del año 2001.

\section{BIBLIOGRAFIA}

Barnett, R.R., T. Akindele, C. Orte \& L. Shephard. 1996. Eosinophilic granulocytes in the epidermis of Oreochromis mossambicus gill filaments studied in situ. J. Fish Biol 49:148-156.

Campbell, T.W \& F. Murru. 1990. An introduction to fish hematology. Compendium of Continuing Education in Veterinary Science 12: 525-533.

Campbell, T.W. 1988. Fish cytology and hematology. Veterinary Clinics of North America - Small Animal Practice 18: 349-364.

Cerda, A. 1994. Valores de referencia de la serie roja en Oncorhynchus mykiss en la piscicultura centro Antuco, Los Angeles, VIII región. Tesis para optar al título de Biólogo Marino. Universidad de Concepción, Chile, 55 págs.

Compagno, L. 1984. FAO species catalogue. Vol. 4. Sharks of the World. An annoted and illustrated catalogue of sharks species known to date. Part. 2. Carcharhiniformes. FAO Fish. Synop. (125) Vol. 4, Pt. 2: 251-655.

Donaldson, E.M. 1981. The pituitary-interrenal axis as an indicator of stress in fish. In: Stress in Fish. (Pikering A.D. Ed.) pp 11-47. Academic Press. London.

ElLis, A.E. 1977. The leucocytes of fish: A review. J.Fish Biol. 14: 453-491.

Fariña, J \& P. OJeda. 1993. Abundance, activity and trophic patterns of the red spotted catshark, Shroederichthys chilensis, on the pacific temperate coast of Chile. Copeia, 2:545-549.

Hantonella ,A. 1998. Interrenal dysfunction in fish from contaminated sites: in vivo and in vitro assessment. Environ. Toxicol. Chem. 17(1): 44-48.

Ishizeki, K., T. NaWA, T. Tachibana \& S. IIDA. 1984. Hemopoietic sites and development of eosinophils granulocytes in the loach, Misgurnus 
Leucocitos de S. chilensis: Valenzuela, A. ET AL

anguillicaudatus. Cell Tissue Res. 235:419-426.

Khan, R. 1977. Blood changes in Atlantic Cod (Gadus morhua) infected with Trypanosoma murmanensis. J. Fish. Res. Board Can. 34: 2193 2196.

Kita, J. \& Y. ITAZAWA. 1989. Release of erythrocytes from the spleen during exercise and splenic constriction by adrenaline infusion in the rainbow trout. Jpn. J. Ichthyol. 36(1):48-52.

Larsson, A.; Johansson-SJöbeck, M. L. \& R. Fange. 1976. Comparative study of some haematological and biochemical blood parameters in fishes from the Skagerrak. J. Fish Biol. 9:425-440.

MahaJan, C.L., Dheer, J.M.S., 1980. An autoradiographic and cytochemical study of erythropoiesis in a fresh water fish, Channa punctatus Bloch J. Fish. Biol. 17, 641-648.

Morillas, J.A. 1981. Tipos celulares y mediciones de las células sanguíneas de pintarroja. Halaelurus chilensis (G.)(Pisces, Chondrichthyes). Bol.Soc.Biol de Concepción. Tomo LII, 103-107.

Morillas, J.A. 1981.Estructura fina de granulocitos y trombocitos de pintarroja, Halaelurus chilensis, (G.)(Pisces, Chondrichthyes). Bol. Soc. Biol de Concepción. Tomo LII, 109-114.

Morillas, J; G. Nascimento, H. Valeria \& R. Khan. 1987. Trypanosoma humboldti n.sp. from the Chilean catshark, Shroederichthys chilensis (Guichenot, 1948). J. Protozool. 34(3):342-344.

Powell, M.D.; G.M. Wright \& J.F. BuRKa. 1990. Eosinophilic granule cells in the gills of rainbow trout, Oncorhynchus mykiss: evidence of migration?. J. Fish Biol. 37: 495-497.
SHERBURNe, S.W. 1974. Ocurrence of both heterophils and neutrophils in the blood of the spiny dogfish, Squalus acanthias. Copeia. 1:259-261.

Valenzuela, A., K, Alveal \& E. Tarifeño. 2002. Respuesta hematológica de truchas (Oncorhynchus mykiss, Walbaum 1792) a estrés hipoxico agudo: Serie roja. Gayana 66(2):255261.

Valenzuela, A., V. Silva \& C. Oyarzún. 1999. Caracterización cualitativa y cuantitativa de células sanguíneas de robalo Eleginoips maclovinus (Valenciennes, 1830)(Pisces, Eleginopsidae) en la desembocadura del río BioBio. Rev. Biol. Mar. Oceanogr. 34(2):261-267.

Wedemeyer, G.A.; B..A. Barton \& D..J. McLeay., 1990. Stress and aclimation.. in: Schreck, C.B., Moyle, P.B. (Eds.), Methods for Fish Biology. Am. Fischeries Soc.

WhaLI, T. 2002. Aproaches to investigate environmental impacts on fish healt. Bull. Eur. Ass. Fish Pathol. 22(2):126- 132.

Wilhelm, .D.F.; G.J. Eble, G.; Kassner.; F.X. Caprario, A.L. DAFré \& M. OHIRA. 1992. Comparative hematology in marine fish. Comp.Biochem and Physiol. 102: 311-321.

Williams, W., E, Beutler., A, Erslev \& M, Lichtman. 1991. Hematology. Fourth edition. Editorial McGraw-hill. United States of America. 1882 pp.

ZвAnyszek, R. \& L. S Sмiтн. 1984. The effect of watersoluble aromatic hydrocarbons on some haematological parameters of rainbow trout, Salmo gairdneri Richardson, during acute exposure. J.Fish Biol. 24,545-552.

Fecha de recepción: 22/04/03

Fecha de aceptación: 04/06/03 